\title{
ON THE GROWTH RATE OF TUNNEL NUMBER OF KNOTS
}

\author{
TSUYOSHI KOBAYASHI AND YO'AV RIECK
}

\begin{abstract}
Given a knot $K$ in a closed orientable manifold $M$ we define the growth rate of the tunnel number of $K$ to be $g r_{t}(K)=$ $\limsup _{n \rightarrow \infty} \frac{t(n K)-n t(K)}{n-1}$. As our main result we prove that the Heegaard genus of $M$ is strictly less than the Heegaard genus of the knot exterior if and only if the growth rate is less than 1 . In particular this shows that a non-trivial knot in $S^{3}$ is never asymptotically super additive. The main result gives conditions that imply falsehood of Morimoto's Conjecture.
\end{abstract}

\section{INTRODUCTION}

This paper is concerned with the asymptotic behavior of the tunnel number of knots in closed 3-manifolds under repeated connected sum operation. Let $t(K)$ be the tunnel number of a knot $K$ (for definitions see Section (2). It is well known that for any pair of knots $K_{1}, K_{2}$ the following inequality holds:

$$
t\left(K_{1} \# K_{2}\right) \leq t\left(K_{1}\right)+t\left(K_{2}\right)+1 .
$$

For convenience we denote $\#_{i=1}^{n} K$, the connected sum of $n$ copies of $K$, by $n K$. By applying Inequality (1D) repeatedly we obtain:

$$
t(n K) \leq n t(K)+(n-1) .
$$

We define the growth rate of the tunnel number of $K$, denoted by $g r_{t}(K)$, to be:

$$
g r_{t}(K)=\limsup _{m \rightarrow \infty} \frac{t(m K)-m t(K)}{m-1} .
$$

Remarks 1.1. (1) Inequality (II) implies that $\frac{t(m K)-m t(K)}{m-1} \leq 1$ for any $m$. Since $t(m K) \geq 0$, we obtain $\frac{t(m K)-m t(K)}{m-1} \geq \frac{-m}{m-1} t(K)$.

Date: July 25, 2018.

The first named author was supported in part by grant in aid JSPS 15540073 . 
Combining the two we see that $-t(K) \leq g r_{t}(K) \leq 1$. Therefore $g r_{t}(K)$ exists and is finite.

(2) We say that a knot $K$ is meridionally small if the meridian of $K$ is not the boundary of an essential surface (i.e., $\pi_{1}$ injective, not boundary parallel surface) embedded in $E(K)$. If $K$ is meridionally small then:

$$
g r_{t}(K) \geq 0 \text {. }
$$

In fact, if $K$ is meridionally small then, by Mor00b and $[\mathrm{KR}$, we have $t(n K) \geq n t(K)$ for any $n$, which implies the above inequality.

(3) If $K$ is a knot in $S^{3}$ then:

$$
g r_{t}(K) \geq-\frac{2}{3} t(K)-1 .
$$

In fact, Scharlemann-Schultens SS00 (quoting Kwong [Kwo94) have the inequality $t(n K) \geq \frac{n}{3} t(K)-(n-1)$. This implies:

$$
\begin{aligned}
\frac{t(n K)-n t(K)}{n-1} & \geq \frac{(n / 3) t(K)-(n-1)-n t(K)}{n-1} \\
& =\frac{(-2 n / 3) t(K)-(n-1)}{n-1} .
\end{aligned}
$$

Taking limit gives the above inequality.

Note that for any knot $K$, the Heegaard genus of the knot exterior $E(K)$, denoted $g(E(K))$, is equal to $t(K)+1$, and it is at least the Heegaard genus of the ambient manifold $M$, denote $g(M)$. In this paper, we prove the following (for definitions see Section [2):

Theorem 1.2. Let $K$ be a knot in a closed, orientable 3-manifold $M$ such that the $g(M)<g(E(K))$. Suppose $K$ is a genus $t(K)$, n-bridge knot (i.e., $n$ is the bridge index of $K$ with respect to Heegaard surfaces of genus $t(K)(=g(E(K))-1))$. Then $\operatorname{gr}_{t}(K) \leq \frac{n-1}{n}$. In particular, for sufficiently large $n$ equality does not hold in Inequality (2).

Note that the assumption of Theorem 1.2 holds trivially for any knot in $S^{3}$.

Suppose that a knot $K$ is isotopic onto a genus $g$ Heegaard surface $\Sigma \subset M$. (We are not assuming that $\Sigma$ is of minimal genus.) By connecting a spine of one of the handlebodies complementary to $\Sigma$ to $K$ via a vertical arc it is easy to see that $t(K) \leq g$. As a consequence of Theorem 1.2 we have the following: 
Corollary 1.3. Let $M$ be a manifold, $\Sigma \subset M$ a genus $g$ Heegaard surface and $K \subset \Sigma$ a knot. If $t(K)=g$, then $\operatorname{gr}_{t}(K) \leq 0$.

Proof. By a slight isotopy of $K \subset \Sigma$ it is easy to see that $K$ is 1-bridge with respect to $\Sigma$. We can therefore apply Theorem 1.2 to obtain the corollary.

Let $T_{p, q}$ be a non-trivial torus knot. Note that $T_{p, q}$ embeds in a genus 1 Heegaard surface of $S^{3}$ and $t\left(T_{p, q}\right)=1$. Hence, by Corollary [1.3. $g r_{t}\left(T_{p, q}\right) \leq 0$. On the other hand, it is known that torus knots are meridionally small. Therefore by $(2)$ of Remarks $1.1 g r_{t}\left(T_{p, q}\right) \geq 0$. As a conclusion, we have that $g r_{t}\left(T_{p, q}\right)=0$.

Similarly, given any Heegaard surface $\Sigma \subset M$ and a knot $K \subset$ $\Sigma$ fulfilling the hypotheses of Corollary 1.3, by performing any Dehn surgery for which the boundary of the meridian of the attached solid torus intersects each component of the boundary of $\Sigma \cap E(K)$ exactly once, we get $M^{\prime}, \Sigma^{\prime}$ and $K^{\prime}$ also fulfilling the hypotheses of Corollary 1.3 (see, for example, Rie00). By Hatcher Hat82 after excluding a finite set, $K^{\prime}$ is known to be meridionally small. Therefore we obtain infinitely many knots $K^{\prime}$ with $g r_{t}\left(K^{\prime}\right)=0$.

Suppose, particularly, that $K$ is isotopic onto some minimal genus Heegaard surface. Such knots are called good in Rie00 where it was shown that either $g(M)=t(K)$ or $g(M)=t(K)+1$. For such knots, Corollary 1.3 implies that if $g(M)=t(K)$, then $g r_{t}(K) \leq 0$. In contrast to this, in case when $g(M)=t(K)+1$ (equivalently, $g(M)=g(E(K))$ ) we have the following. (Note that if $g(E(K))=g(M)$ then $K$ is necessarily isotopic onto a minimal genus Heegaard surface of $M$.)

Theorem 1.4. Let $K$ be a knot in a closed, orientable 3-manifold $M$ such that $g(M)=g(E(K))$. Then for all $n, t(n K)=n t(K)+n-1$. In particular, $g r_{t}(K)=1$, and $\lim _{m \rightarrow \infty} \frac{t(m K)-m t(K)}{m-1}$ exists.

So we see that the growth rate is 1 if and only if $g(M)=g(E(K))$, i.e., if and only if $K$ is a core of a handlebody in a minimal genus Heegaard splitting of $M$.

In the rest of this section we describe a relationship between Theorem 1.2 and Morimoto's Conjecture, which is concerned with the super additive phenomenon of the tunnel number of knots. In Mor00a Morimoto conjectured that $t\left(K_{1} \# K_{2}\right) \leq t\left(K_{1}\right)+t\left(K_{2}\right)$ if and only if $K_{1}$ or $K_{2}$ admits a primitive meridian (for definitions, see Subsection 2.3). Here we note the well-known fact that if $K_{1}$ or $K_{2}$ admits a primitive meridian, then $t\left(K_{1} \# K_{2}\right) \leq t\left(K_{1}\right)+t\left(K_{2}\right)$ (see Proposition 2.1] in section 2). 
In this paper we show that the growth rate can furnish a method for disproving Morimoto's Conjecture. For this purpose, we first demonstrate the following theorem with assuming Theorem [1.2, and Proposition 2.1.

Theorem 1.5. If there exists a knot $K \subset S^{3}$ so that neither $K$ nor $2 K(=K \# K)$ admits a primitive meridian then Morimoto's Conjecture is false.

Remark. The following proof shows that Theorem [1.5] still holds for knots $K \subset M$ in an arbitrary manifold, provided that $g(E(K))>$ $g(M)$.

Proof. By Theorem 1.2 for some $n$ we have that

$$
t(n K)<n t(K)+(n-1) .
$$

From now on, take $n \geq 2$ to be the minimal $n$ with that property.

If $n=2$ then two copies of $K$ provide the desired counterexample. Thus we may assume that $n>2$. Consider the knots $K_{1}=2 K$ and $K_{2}=(n-2) K$. Note that $K_{1}$ does not admit a primitive meridian by assumption and $K_{2}$ does not admit a primitive meridian by Proposition 2.1 and the minimality of $n$.

We have:

(1) $t\left(K_{1}\right)=2 t(K)+1$ (by minimality of $\left.n\right)$,

(2) $t\left(K_{2}\right)=(n-2) t(K)+(n-3)$ (by minimality of $n$ ), and-

(3) $t\left(K_{1} \# K_{2}\right)=t(n K)<n t(K)+(n-1)=t\left(K_{1}\right)+t\left(K_{2}\right)+1$.

Hence $K_{1}$ and $K_{2}$ provide a counterexample to Morimoto's Conjecture.

The following corollary follows from Theorem 1.5, and Lemma 3.2 in section 3 .

Corollary 1.6. If there exists a knot in $S^{3}$ with growth rate grater than $1 / 2$ then Morimoto's Conjecture is false.

Remark. The following proof shows that Corollary 1.6] still holds for knots $K \subset M$ in an arbitrary manifold, provided that $g(E(K))>$ $g(M)$.

Proof. Let $K \subset S^{3}$ be a knot with $g r_{t}(K)>\frac{1}{2}$. If $K$ admits a primitive meridian, then by Proposition $2.1 t(K \# q K) \leq t(K)+t(q K)$ for any positive integer $q$. This implies $t(n K) \leq n t(K)$ for any $n$, and consequently we have $g r_{t}(K) \leq 0$, a contradiction. If $K \# K$ admits a primitive meridian, then for any $q$ we have that $t(2 K \# q K) \leq t(2 K)+t(q K)$, and by Lemma 3.2. $g r_{t}(K) \leq \frac{1}{2}$, a contradiction; hence neither $K$ nor 
$2 K$ admits a primitive meridian and Morimoto's Conjecture is false by Theorem 1.5.

We conclude this section with some open questions related to the above; in all these questions we assume that $g(M)<g(E(K))$. Let $g$ denote the Heegaard genus of $E(K)$.

For the definitions of genus $g, n$-bridge presentation of a knot, and $(g, n)$-knot in a closed 3-manifold, see Subsection 2.4. It is well known (Proposition 2.4 ) that $K$ admits a primitive meridian if and only if $K$ is a $(t(K), 1)$-knot, that is, $K$ has bridge index one with respect to some genus $t(K)$ Heegaard surface of $M$. In the proof of Theorem 1.2 we generalize this and see that:

Proposition 1.7. If $K$ is a $(t(K), n)$-knot then either $n K$ admits a primitive meridian or $t(n K)<n t(K)+n-1$ (possibly both).

We note that an interesting outcome of Proposition 1.7 is the case when $t(n K)=n t(K)+n-1$ with $n K$ admitting a primitive meridian (in Section 4 we will see such phenomenon indeed occurs for $n=2$ ). About the converse we ask:

Question 1.8. Suppose $t(n K)=n t(K)+n-1$ and that $n K$ admits a primitive meridian. Does $K$ admit a genus $t(K)$, $n$-bridge presentation? Specifically, suppose $t(2 K)=2 t(K)+1$ and that $2 K$ admits a primitive meridian. Does $K$ have a genus $t(K)$, 2-bridge presentation?

Next we ask:

Question 1.9. Does there exist a knot $K$ so that $K$ is a $(t(K), n)$ knot for $n$ at least 3? Specifically, does there exist a tunnel number 1 knot in $S^{3}$ that has bridge index greater than 2 with respect to the genus 1 Heegaard splitting of $S^{3}$ ?

Remark. If there exists a knot for which the answers to Question 1.8 (the case $n=2$ ) and Question 1.9 are both "yes" then neither $K$ nor $2 K$ admits a primitive meridian. Therefore, by Theorem 1.5. Morimoto's Conjecture is false.

Question 1.10. What is the spectrum of growth rates?

We note that it is known that we can construct arbitrarily high degeneration of tunnel number of knots under connected sum (see Kob94), nevertheless the following question is still open.

Question 1.11. Does there exist a knot with negative growth rate? If so, can the growth rate of knots be arbitrarily negative?

In light of Corollary 1.6 we ask: 
Question 1.12. Is there a knot with growth rate greater than $1 / 2$ and less than 1?

We know little about the properties of the sequence $\frac{t(m K)-m t(K)}{m-1}$. In particular:

Question 1.13. Is the sequence $\frac{t(m K)-m t(K)}{m-1}$ eventually monotonous? Does $\lim _{m \rightarrow \infty} \frac{t(m K)-m t(K)}{m-1}$ exist?

Question 1.14. Given a knot $K$, is $t(n K) \leq t((n+1) K)$ ?

We remark that while a positive answer would give bounds on the behavior of the elements $\frac{t(n K)-n t(K)}{n-1}$, it will not suffice to show that the limit exists.

Acknowledgements: We thank Chaim Goodman-Strauss and Mark Johnson for helpful conversations.

\section{Preliminaries}

Throughout this paper we work in the smooth category. We always assume our manifold to be compact and orientable. For standard notion in 3-manifold topology we refer the reader to Hem76] or Jac80.

2.1. Amalgamation of Heegaard splittings. A 3-manifold $C$ is a compression body if there is a closed connected surface $F$ such that $C$ is obtained from $F \times[0,1]$ by attaching 2-handles along mutually disjoint simple closed curves in $F \times\{1\}$ and capping off the resulting 2-sphere boundary components which are disjoint from $F \times\{0\}$ by 3handles. The boundary component of $C$ corresponding to $F \times\{0\}$ is denoted $\partial_{+} C$. Then $\partial_{-} C=\partial C \backslash \partial_{+} C$. A compression body $C$ is called a handlebody if $\partial_{-} C=\emptyset$. A compressing disk $D \subset C$ for $\partial_{+} C$ is called a meridian disk of the compression body $C$.

By extending the cores of the 2-handles in the definition of the compression body $C$ vertically to $F \times[0,1]$ we obtain a collection of mutually disjoint meridian disks of $C$, say $\mathcal{D}$, such that the manifold obtained from $C$ by cutting along $\mathcal{D}$ is homeomorphic to a union of $\partial_{-} C \times[0,1]$ and a (possibly empty) collection of 3 -balls. This gives a dual description of the compression body, that is, a connected 3 -manifold $C$ is a compression body if there exists a (not necessarily connected and possibly empty) closed surface $\mathcal{F}$, without 2 -sphere components, and a (possibly empty) collection of 3-balls $\mathcal{B}$ such that $C$ is obtained from $\mathcal{F} \times[0,1] \cup \mathcal{B}$ by attaching 1 -handles to $\mathcal{F} \times\{0\} \cup \partial \mathcal{B}$. We note that $\partial_{-} C$ is the surface corresponding to $\mathcal{F} \times\{1\}$.

Let $N$ be a compact 3-manifold and $F_{1}, F_{2}$ a partition of the components of $\partial N$. We say that a decomposition $C_{1} \cup_{\Sigma} C_{2}\left(\right.$ or $\left.C_{1} \cup C_{2}\right)$ is 
a Heegaard splitting of $\left(N ; F_{1}, F_{2}\right)$ (or of $\left.N\right)$ if it satisfies the following conditions:

(1) $C_{i}(i=1,2)$ is a compression body in $N$ such that $\partial_{-} C_{i}=F_{i}$,

(2) $C_{1} \cup C_{2}=N$, and-

(3) $C_{1} \cap C_{2}=\partial_{+} C_{1}=\partial_{+} C_{2}=\Sigma$.

The surface $\Sigma$ is called a Heegaard surface of $\left(N ; F_{1}, F_{2}\right)$ (or $\left.N\right)$. The genus of $\Sigma$ is called the genus of the splitting and the genus of the minimal genus Heegaard splitting for $N$ is called the Heegaard genus of $N$, denoted $g(N)$.

Let $\Sigma_{1} \subset M_{1}$ and $\Sigma_{2} \subset M_{2}$ be two Heegaard surfaces for 3-manifolds $M_{1}$ and $M_{2}$. Suppose that a component $T_{1}$ of $\partial M_{1}$ and a component $T_{2}$ of $\partial M_{2}$ are homeomorphic. Let $M$ be a manifold obtained from $M_{1}$ and $M_{2}$ by identifying $T_{1}$ and $T_{2}$ by a homeomorphism. Then we can obtain a Heegaard surface for $M$, say $\Sigma$, from $\Sigma_{1}$ and $\Sigma_{2}$ by collapsing the product region adjacent to $T_{1}$ and $T_{2}$ as in Figure 1 . We call $\Sigma$ the amalgamation of $\Sigma_{1}$ and $\Sigma_{2}$ along $T$, where $T$ is the image of $T_{1}=T_{2}$ in $M$. For details, see [Sch93, where it was shown that:

$$
g(\Sigma)=g\left(\Sigma_{1}\right)+g\left(\Sigma_{2}\right)-g(T)
$$

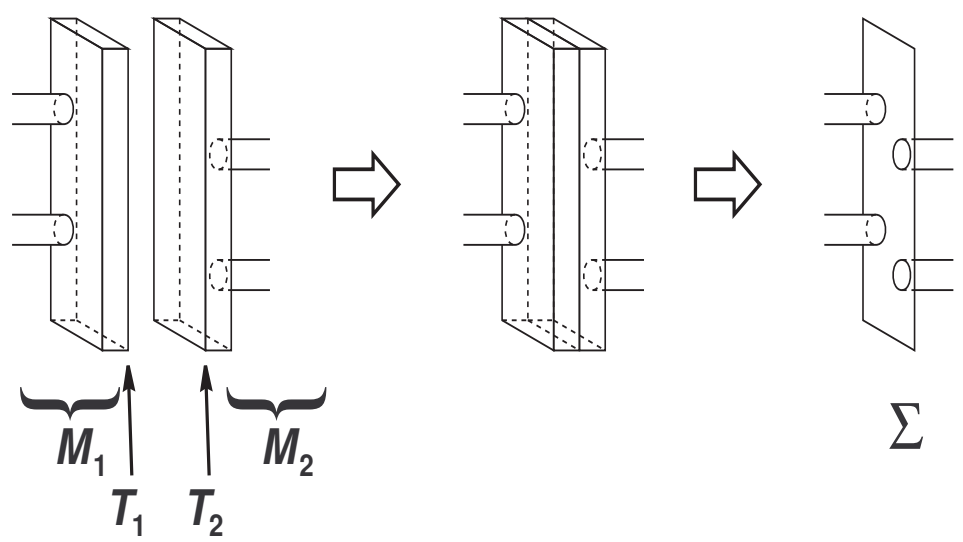

Figure 1

2.2. Tunnel number. Let $M$ be a compact orientable 3 -manifold. By a knot $K$ we mean a smooth embedding of $S^{1}$ into $M$. For a knot $K \subset$ $M$, let $E(K)$ denote the exterior of $K$, i.e., $E(K)=M \backslash \operatorname{int}(N(K))$. It is an easy consequence of the existence of smooth structures on $E(K)$ that there exists a collection of properly embedded arcs $\tau$ such that $\operatorname{cl}(E(K) \backslash N(\tau))$ is a handlebody. Such a collection $\tau$ is called a 
tunnel system. The tunnel number of $K$, denoted $t(K)$, is the minimal number of arcs required for tunnel systems. Then $t(K)$ is related to the Heegaard genus of $E(K)$ by the equation $t(K)=g(E(K))-1$. Given two knots $K_{1} \subset M_{1}$ and $K_{2} \subset M_{2}$ the connected sum of $K_{1}$ and $K_{2}$, denoted $K_{1} \# K_{2}$, is a knot in $M_{1} \# M_{2}$ which is obtained by removing from $M_{i}$ a small ball so that $K_{i}$ intersects this ball in a single unknotted arc $(i=1,2)$ and then identifying the boundaries of the punctured manifolds by a homeomorphism under which the intersection of the knots with the boundaries match up. By taking a union of a tunnel system for $K_{1}$, a tunnel system for $K_{2}$ and one extra tunnel on the decomposing sphere we obtain a tunnel system for $K_{1} \# K_{2}$. This gives the Inequality (11) of Section 1.

When equality in Inequality (11) holds we say that the tunnel number of the knots are super additive.

2.3. Primitive meridian. Let $M, K$ be as above. We say that $K$ admits a primitive meridian if there is a minimal genus Heegaard splitting of $E(K)$, say $C \cup_{\Sigma} V$ (here $C$ is a compression body and $V$ is a handle body, so that $\left.\partial E(K)=\partial_{-} C\right)$, such that there exists a properly embedded essential annulus $A \subset C$ and a meridian disk $D \subset V$ with the following properties:

(1) $A$ is vertical in $C$ (i.e., one boundary component of $A$ is on $\partial_{+} C$ and the other on $\partial_{-} C$ ) and $A \cap \partial_{-} E(K)$ is a meridian curve of $E(K)$.

(2) $A \cap \Sigma\left(=A \cap \partial_{+} C\right)$ and $D \cap \Sigma(=\partial D)$ intersect transversely (in $\Sigma$ ) in one point.

An important feature of knots admitting a primitive meridian is that they are never super additive (recall Subsection 2.2) when connected sum to other knots. In fact, Propositions 1.3 and 2.1 of [Mor00a] imply the following:

Proposition 2.1. If $K$ admits a primitive meridian then for any knot $K^{\prime}$ we have:

$$
t\left(K \# K^{\prime}\right) \leq t(K)+t\left(K^{\prime}\right) .
$$

In Mor00a Morimoto conjectures that the converse of this is true:

Conjecture 2.2 (Morimoto's Conjecture). If $t\left(K \# K^{\prime}\right) \leq t(K)+$ $t\left(K^{\prime}\right)$, then $K$ or $K^{\prime}$ admits a primitive meridian.

Remark. This conjecture is stated in a different appearance in Mor00a. By Proposition 2.1 of that paper (see Proposition 2.4 below), we can show that the two are equivalent. 
In $[\mathrm{KR}$ the authors give a necessary and sufficient condition for a knot $K$ to admit a primitive meridian. We introduce the result here.

Notation: For a knot $K$, we denote by $\widehat{K}$ the link obtained from $K$ by adding a single simple closed curve parallel to the meridian of $K$; in other words, $\widehat{K}=K \#$ (Hopf link in $S^{3}$ ). For $n \geq 0$ we denote by $K^{(n)}$ the link obtained from $K$ by adding $n$ simple closed curves simultaneously parallel to $n$ disjoint copies of the meridian in $E(K)$ (here we understand $K^{(0)}=K$ and $K^{(1)}=\widehat{K}$ ). The exterior of $\widehat{K}$ is denoted by $\widehat{E(K)}$ and the exterior of $K^{(n)}$ is denoted by $E(K)^{(n)}$. An essential annulus $A$ properly embedded in $\widehat{E(K)}\left(\operatorname{resp} . E(K)^{(n)}\right)$ is called a Hopf spanning annulus if one boundary component of $A$ is a meridian of $K$ and the other a longitudinal curve of $\partial \widehat{E(K)} \backslash \partial E(K)$ (resp. some component of $\partial E(K)^{(n)} \backslash \partial E(K)$ ).

Then the following holds:

Proposition 2.3. A knot $K \subset M$ admits a primitive meridian if and only if there exists a Heegaard splitting $U \cup_{\Sigma} V$ of $\widehat{E(K)}$ which satisfies the following conditions:

(1) The genus of $\Sigma$ equals $g(E(K))$,

(2) There exists a Hopf spanning annulus $A \subset \widehat{E(K)}$ such that $A$ intersects $\Sigma$ transversely in a single simple closed curve that is essential in $A$.

The proof of this proposition can be found in $[\mathrm{KR}$. In this paper we only use the "if" part of this proposition and the idea of its proof is as follows: since $E(K)$ is obtained form $\widehat{E(K)}$ by Dehn filling, any Heegaard surface for $\widehat{E(K)}$ is also a Heegaard surface for $E(K)$. The Heegaard surface for $\widehat{E(K)}$ that is described in the proposition naturally admits a primitive meridian. See Figure 2 .

2.4. Generalized bridge number. Let $U \cup_{\Sigma} V$ be a Heegaard splitting of some 3-manifold $M$, and $K \subset M$ a knot. Following Doll Dol92 we say that $K$ is in genus $g, n$-bridge presentation (with respect to $\Sigma$ ) if $K \cap U$ ( $K \cap V$ resp.) consists of $n$ arcs, and these arcs are simultaneously parallel into $\partial U$ ( $\partial V$ resp.) (The genus is omitted when clear from context). We say that $K$ is a $(g, n)$-knot, or a genus $g, n$-bridge knot, if $K$ admits a genus $g, n$ bridge presentation but does not admit a genus $g, n-1$ bridge presentation for any Heegaard surface of genus $g$.

Then we have the following characterization of knots with primitive meridian (see Proposition 2.1 of [Mor00a]): 


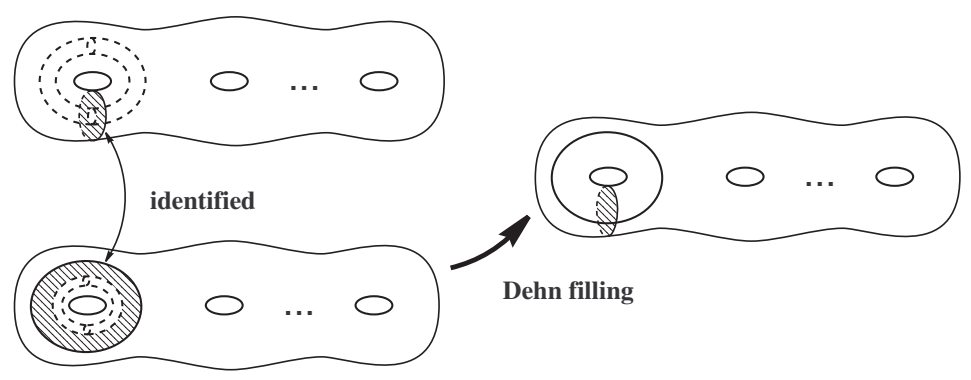

Figure 2

Proposition 2.4. Let $K, M$ be as above. Then $K$ admits a primitive meridian if and only if $K$ is a $(t(K), 1)$-knot (or, $(g(E(K))-1,1)$ knot).

\section{THE PROOF}

In this section we prove Theorems [1.2, and 1.4. For the proof of Theorem 1.2, we first prepare two lemmas:

Lemma 3.1. Let $K$ be a knot in a closed orientable 3-manifold $M$. Suppose there exists a positive number $n$ so that $t(n K)<n t(K)+n-1$. Then $g r_{t}(K) \leq \frac{n-1}{n}$.

Proof. Fix a non-negative integer $m$. Write $m$ as $p n+l$, with $0 \leq l<n$. We have inequalities below. (Notes: (1) In the following we take $t(0 K)$ to be 0 . (2) In one line of the following lines we need to treat the cases $l=0, l>0$ separately. We can deal with both cases by introducing a variable $q$ such that $q=p$ if $l>0$, and $q=p-1$ if $l=0$. (3) Some lines contain explanations in [brackets]; there by Equation (11) we mean Equation (11) of Section 11) 


$$
\begin{aligned}
& t(m K)=t((n p+l) K) \\
& \leq t(n K)+t([(p-1) n+l] K)+1 \quad \text { [By Equation (10)] } \\
& \leq 2 t(n K)+t([(p-2) n+l] K)+2 \quad \text { [By Equation (II) }] \\
& \text {. . } \\
& \leq p t(n K)+t(l K)+q \\
& \leq p(t(n K))+l t(K)+(l-1)+p \\
& \leq p(n t(K)+n-2)+l t(K)+(l-1)+p \\
& \text { [since } t(n K)<n t(K)+n-1 \text { by assumption] } \\
& \leq(n p+l) t(K)+(n p+l-1)-p \\
& =m t(K)+(m-1)-p \text {. }
\end{aligned}
$$

We conclude that the growth rate fulfills the inequality $\frac{t(m K)-m t(K)}{m-1} \leq$ $\frac{m-1-p}{m-1}$. In the limit $m \approx n p$ and we get the bound $\frac{(n-1) p}{n p}=\frac{(n-1)}{n}$ as desired. This completes the proof of Lemma 3.1.

Lemma 3.2. Let $K$ be a knot in a closed orientable 3-manifold $M$. Suppose that there exists a positive integer $n$ so that for any positive integer $q$ we have that $t((n+q) K) \leq t(n K)+t(q K)$. Then $\operatorname{gr}_{t}(K) \leq$ $\frac{n-1}{n}$.

Proof. Again let $m$ be a positive integer and write $m$ as $p n+l$, with $0 \leq l<n$. By repeatedly applying the assumption of Lemma 3.2. and then applying Equation (2) in section 1 twice we have: (Note: When we apply Equation (2) to $t(l K)$ there are two possible results, depending on whether $l=0$ or not; these are treated by using $\max \{l-1,0\}$. Eventually we arrive at a bound that is valid in both cases.)

$$
\begin{array}{rlr}
t(m K) & =t((n p+l) K) & \\
& \leq t(n K)+t([(p-1) n+l] K) & \text { [by assumption] } \\
& \leq 2 t(n K)+t([(p-2) n+l] K) & \text { [by assumption] } \\
& \ldots & \\
& \leq p t(n K)+t(l K) & \\
& \leq p[n t(K)+(n-1)]+t(l K) & \text { [by assumption] } \\
& \leq p[n t(K)+(n-1)]+l t(K)+\max \{l-1,0\} \\
& \leq(n p+l) t(K)+(n p+\max \{l-1,0\})-p \\
& \leq m t(K)+m-p .
\end{array}
$$


Note that if $l \neq 0$ we actually get the bound $m t(K)+(m-1)-p$, which is exactly the bound we obtained in the proof of Lemma 3.1. Since the constant +1 vanishes in the limit, we complete the proof of Lemma 3.2 exactly as above.

Proof of Theorem 1.2 and Proposition 1.7. Let $K \subset M, n$ be as in Theorem 1.2. Suppose that $n=1$. By Proposition 2.4, $K$ admits a primitive meridian. Hence, by applying Proposition 2.1 repeatedly, we see that for any positive integer $m$ we have that $t(m K) \leq m t(K)$. Hence $\operatorname{gr}_{t}(K) \leq 0=\frac{1-1}{1}$; this gives Theorem 1.2. Hence for the remainder of the proof we assume that $n \geq 2$.

If $t(n K)<n t(K)+(n-1)$ then by Lemma 3.1 we have the conclusion of Theorem 1.2. Hence for the remainder of the proof we may assume that $t(n K)=n t(K)+(n-1)$, and will show that $n K$ admits a primitive meridian. (Note that this proves Proposition 1.7.) Let $U \cup_{\Sigma} V$ be a genus $t(K)$ Heegaard splitting of $M$ with respect to which $K$ admits an $n$ bridge presentation. Isotope $K$ to such position. Hence $K \cap U$ ( $K \cap V$ resp.) consists of $n$ boundary parallel arcs. Then $E(K) \cap U$ $\left(E(K) \cap V\right.$ resp.) is a genus $t(K)+n$ handlebody, say $U^{\prime}$ ( $V^{\prime}$ resp.) such that $U^{\prime} \cap \partial E(K)\left(V^{\prime} \cap \partial E(K)\right.$ resp.) is a collection of $n$ disjoint simultaneously boundary compressible annuli in $\partial U^{\prime}\left(\partial V^{\prime}\right.$ resp.) as in Figure 3 ; these annuli are meridional in $\partial E(K)$.
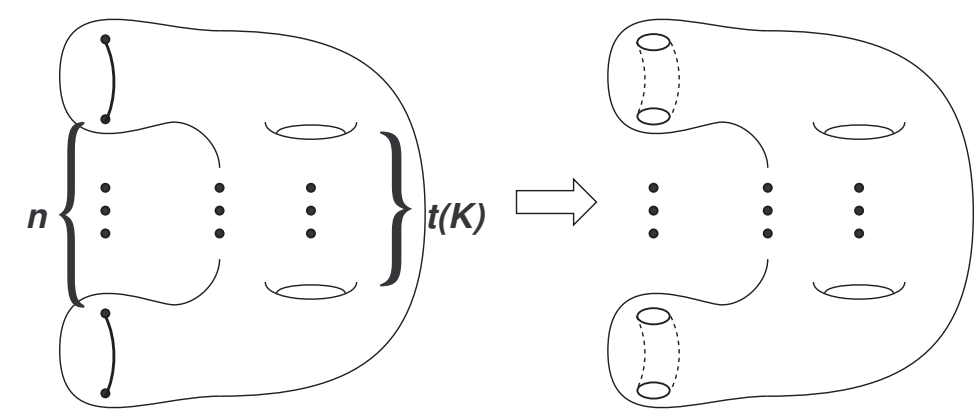

Figure 3

Recall from Subsection 2.3 the notation $K^{(n)}$.

Claim. $E(K)^{(n)}$ admits a genus $t(K)+n$ Heegaard splitting, say $\widetilde{U} \cup_{\widetilde{\Sigma}} \widetilde{V}$, such that $\widetilde{\Sigma}$ intersects a Hopf spanning annulus in a single simple closed curve which is essential in the annulus. 
Proof. Let $A^{(n)}$ be an annulus with $n$ holes. Then let $l^{+}, l^{-}$be the boundary components of the annulus, and let $l_{1}, \cdots, l_{n}$ be the boundary components corresponding to the punctures.

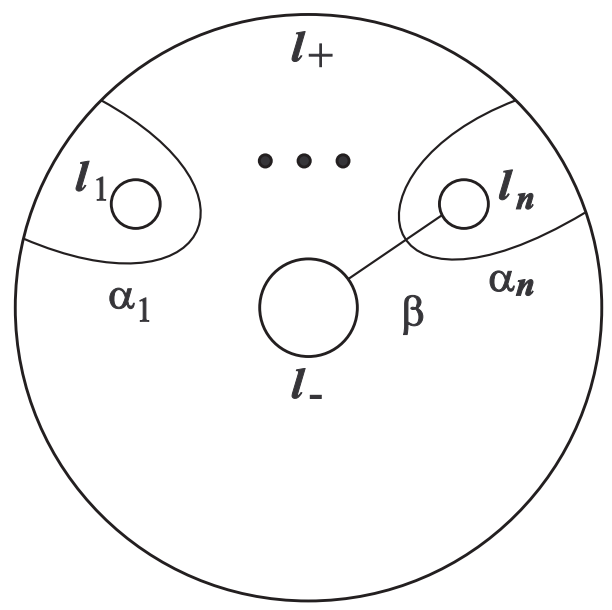

Figure 4

We note that $E(K)^{(n)}$ can be represented as a manifold obtained from $E(K)$ by attaching $A^{(n)} \times S^{1}$ by a homeomorphism $h: \partial E(K) \rightarrow$ $l_{+} \times S^{1}$ that maps a meridian curve to $\{p t\} \times S^{1}$. Let $\alpha_{1}, \cdots, \alpha_{n}$ be arcs properly embedded in $A^{(n)}$ as in Figure 4 . Note that the annuli $\cup_{i=1}^{n}\left(\alpha_{i} \times S^{1}\right)$ cut $A^{(n)}$ into $n+1$ pieces, say $T_{0}, T_{1}, \cdots, T_{n}$ each of which is homeomorphic to (annulus) $\times S^{1}$ (or (torus) $\times[0,1]$ ), where $l_{-} \times S^{1} \subset$ $T_{0}$ and for each $i, l_{i} \times S^{1} \subset T_{i}$. Recall that $\partial E(K) \cap U^{\prime}(\partial E(K) \cap$ $V^{\prime}$ resp.) consists of $n$ annuli. Since $h$ maps a meridian curve to $\{p t\} \times S^{1} \subset l_{+} \times S^{1}$, we may suppose, by deforming $h$ by an isotopy if necessary, that $h\left(E(K) \cap U^{\prime}\right)=\cup_{i=1}^{n}\left(T_{i} \cap\left(l_{+} \times S^{1}\right)\right)$ (hence, $h(\partial E(K) \cap$ $\left.\left.V^{\prime}\right)=T_{0} \cap\left(l_{+} \times S^{1}\right)\right)$. Then let $\widetilde{U}=U^{\prime} \cup_{h}\left(\cup_{i=1}^{n} T_{i}\right)$ and $\widetilde{V}=V^{\prime} \cup_{h} T_{0}$.

By Figures 5 and 6 we have that $\widetilde{U}$ and $\widetilde{V}$ are compression bodies of genus $t(K)+n$, and $\widetilde{U} \cap \widetilde{V}=\partial_{+} \widetilde{U}=\partial_{+} \widetilde{V}(=\widetilde{\Sigma})$. Hence $\widetilde{U} \cup_{\widetilde{\Sigma}} \widetilde{V}$ is a genus $t(K)+n$ Heegaard splitting of $E(K)^{(n)}$. Let $\beta$ be the arc properly embedded in $A^{(n)}$ as in Figure 4 . Note that $\beta \times S^{1}$ gives a Hopf spanning annulus in $E(K)^{(n)}$ and it is directly observed that it intersects the Heegaard surface $\widetilde{\Sigma}$ in a single simple closed curve corresponding to $\left(\alpha_{n} \cap \beta\right) \times S^{1}$. This gives the conclusion of the claim.

Note that $\widehat{E(n K)}$ is obtained from $E(K)^{(n)}$ and $n-1$ copies of $E(K)$ by identifying $l_{1} \times S^{1}, \cdots, l_{n-1} \times S^{1}$ and the boundary components of the copies of $E(K)$ by a homeomorphism which takes each $\{p t\} \times S^{1} \subset$ 


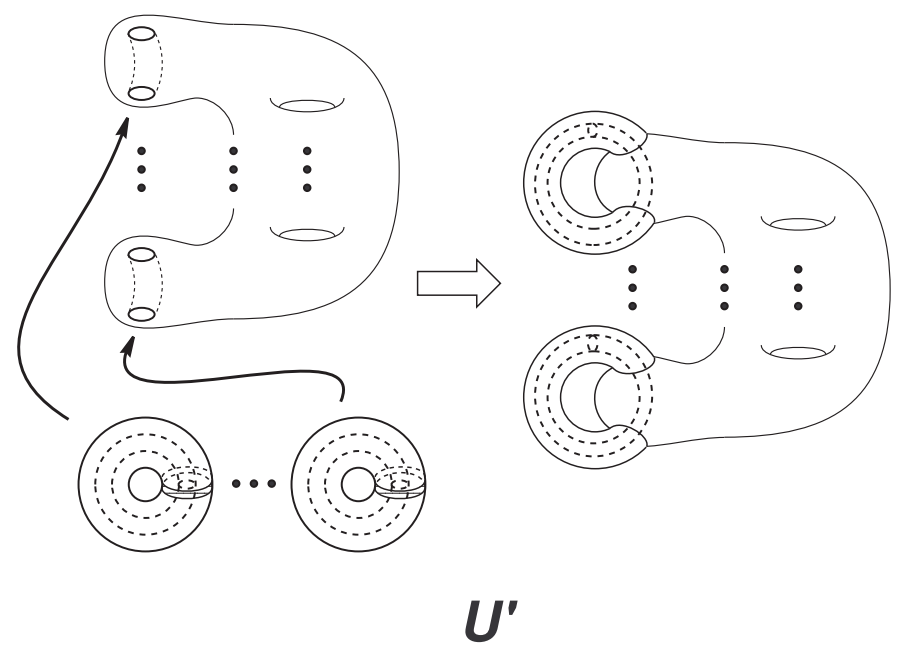

Figure 5

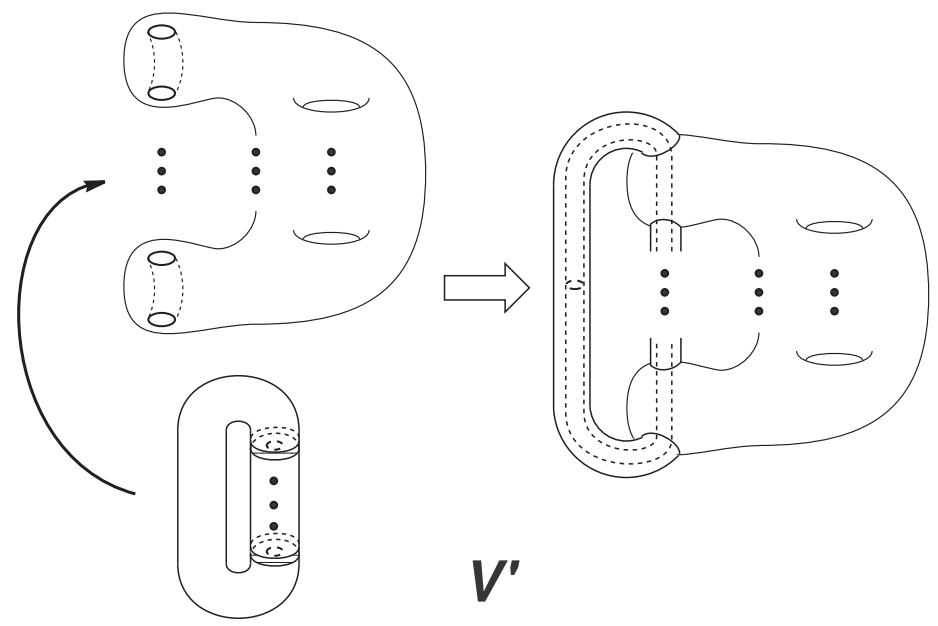

Figure 6

$l_{i} \times S^{1}$ to a meridian curve. Let $Q$ be the Heegaard surface of $\widehat{E(n K)}$ obtained from $\widetilde{\Sigma}$ and a minimal genus Heegaard surface for each of the copies of $E(K)$ by amalgamations. By Equation (4) of Subsection 2.1] the genus of $Q$ fulfills: 


$$
\begin{aligned}
g(Q) & =(t(K)+n)+(n-1) g(E(K))-(n-1) \\
& =t(K)+n+(n-1)(g(E(K))-1) \\
& =t(K)+n+(n-1) t(K) \\
& =n(t(K)+1) \\
& =n g(E(K)) .
\end{aligned}
$$

Recall that we assumed that $t(n K)=n t(K)+(n-1)$ (equivalently $g(E(n K))=n g(E(K)))$ at the beginning of this proof. Hence we have $g(Q)=g(E(n K))$. On the other hand $Q$ is a Heegaard surface for $\widehat{E(n K)}$; hence $g(\widehat{E(n K)}) \leq g(E(n K))$. Since every Heegaard surface of $\widehat{E(n K)}$ is naturally a Heegaard surface of $E(n K)$, we see that $g(\widehat{E(n K)}) \geq g(E(n K))$. Hence we have $g(\widehat{E(n K)})=g(E(n K))$. The amalgamation above does not effect the annulus $\beta \times S^{1}$ and its intersection with $\widetilde{\Sigma}$. Hence by Proposition 2.3 we see that $n K$ admits a primitive meridian. Then by Proposition 2.1 we see that $t((n+q) K) \leq$ $t(n K)+t(q K)$ for any $q$. Then by Lemma 3.2 we see that $g r_{t}(K) \leq \frac{n-1}{n}$.

This completes the proof of Theorem 1.2

Remark. The arguments in the proof of Theorem 1.2 work for a bridge number $n$ with respect to a Heegaard surface of genus $t(K)-k$ for $k>0$. However, it is elementary to show (see, for example, Figure 4 of Kob01) that we can obtain a $(t(K), n-k)$ bridge presentation from the $n$-bridge presentation. Hence we can show that $\operatorname{gr}_{t}(K) \leq \frac{n-k-1}{n-k}$ by Theorem 1.2. Note that $\frac{n-k-1}{n-k}<\frac{n-1}{n}$. This shows that using our techniques, the best estimate of the growth rate is obtained by using the bridge index with respect to genus $t(K)$ Heegaard surfaces.

For the proof of Theorem 1.4, we prepare three lemmas.

Let $\ell$ be a knot in a handlebody $V$. We say that $\ell$ is primitive in $V$ if there is a meridian disk $D$ of $V$ such that $D$ cuts off a solid torus, such that $\ell$ is a core curve of the solid torus.

Lemma 3.3. Let $K$ be a knot in a closed, orientable 3-manifold $M$. Then the equality $g(M)=g(E(K))$ holds if and only if there exists a minimal genus Heegaard splitting $V \cup_{\Sigma} W$ of $M$ such that $K \subset V$, and $K$ is primitive in $V$.

The proof is easy, and we omit it.

Lemma 3.4. Let $K$ be a knot in a closed, orientable 3-manifold $M$. Then the equality $g(M)=g(E(K))$ holds if and only if there exists a minimal genus Heegaard splitting $V \cup_{\Sigma} W$ of $M$ such that $K \subset \Sigma$, 
and there is a meridian disk $D$ of $V$ such that $\partial D$ and $K$ intersects transversely (in $\Sigma$ ) in one point.

Proof. Only if part: Suppose $g(M)=g(E(K))$. By Lemma 3.3, there exists a minimal genus Heegaard splitting $V \cup_{\Sigma} W$ of $M$ such that $K \subset V$, and $K$ is primitive in $V$, i.e., there is a meridian disk $D^{\prime}$ of $V$ such that $D^{\prime}$ cuts off a solid torus $T$ such that $K$ is a core curve of $T$. Then we can isotope $K$ so that $K \subset \partial T$, and $K \cap D^{\prime}=\emptyset$ (hence $K \subset \Sigma)$. Note that $K$ is a longitude of $T$ and this shows that we take a meridian disk $D$ of $V$ such that $\partial D$ and $K$ intersects transversely in one point and that $D \cap D^{\prime}=\emptyset$ (hence $D$ is a meridian disk of $V$ ).

If part: Suppose that $K \subset \Sigma$ and there is a meridian disk $D$ of $V$ such that $\partial D$ and $K$ intersects transversely in one point. Let $N(K \cup$ $D, V)$ be a regular neighborhood of $K \cup D$ in $V$, and $D^{\prime}$ the frontier of $N(K \cup D, V)$ in $V$. It is directly observed that $N(K \cup D, V)$ is a solid torus such that $K$ is a longitude of $N(K \cup D, V)$, and that $D^{\prime}$ is a meridian disk of $V$ which cuts off $N(K \cup D, V)$. Note that $K$ can be isotoped to a core curve of $N(K \cup D, V)$ by an isotopy not affecting $D^{\prime}$. Hence by Lemma 3.3. we see that $g(M)=g(E(K))$.

This completes the proof of Lemma 3.4

Lemma 3.5. Let $K_{i}(i=1,2)$ be knots in a closed, orientable 3manifolds $M_{i}$. Suppose that $g\left(M_{1}\right)=g\left(E\left(K_{1}\right)\right)$, and $g\left(M_{2}\right)=g\left(E\left(K_{2}\right)\right)$ hold. Then we have $g\left(M_{1} \# M_{2}\right)=g\left(E\left(K_{1} \# K_{2}\right)\right)$.

Proof. By Lemma 3.4 there exist minimal genus Heegaard splittings $V_{i} \cup_{\Sigma_{i}} W_{i}$ of $M_{i}(i=1,2)$ such that $K_{i} \subset \Sigma_{i}$, and there is a meridian disk $D_{i}$ of $V_{i}$ such that $\partial D_{i}$ and $K_{i}$ intersects transversely in one point. Then take a 3 -ball $B_{i}$ in $M_{i}$ such that $\Sigma_{i} \cap B_{i}$ is a disk properly embedded in $B_{i}, K_{i} \cap B_{i}$ is an arc properly embedded in $\Sigma_{i} \cap B_{i}$, and $B_{i} \cap D_{i}=\emptyset$. Then we identify the boundaries of $\operatorname{cl}\left(M_{1} \backslash B_{1}\right)$, and $\operatorname{cl}\left(M_{2} \backslash B_{2}\right)$ by a homeomorphism which identifies $\partial\left(\Sigma_{1} \backslash B_{1}\right)$ with $\partial\left(\Sigma_{2} \backslash B_{2}\right)$ and $\partial\left(K_{1} \backslash B_{1}\right)$ with $\partial\left(K_{2} \backslash B_{2}\right)$ to obtain a connected sum $K_{1} \# K_{2}$. We note that the image of $\operatorname{cl}\left(\Sigma_{1} \backslash B_{1}\right) \cup \mathrm{cl}\left(\Sigma_{2} \backslash B_{2}\right)$ is a minimal genus Heegaard surface of $M_{1} \# M_{2}$ (Proposition II.10 of [Jac80]), and $K_{1} \# K_{2}$ is contained in the Heegaard surface. Note also that $D_{1}$ survives in the Heegaard splitting as a meridian disk, and $\partial D_{1}$ intersects $K_{1} \# K_{2}$ transversely in one point. Hence by Lemma 3.4, we see that $g\left(M_{1} \# M_{2}\right)=g\left(E\left(K_{1} \# K_{2}\right)\right)$.

This completes the proof of Lemma 3.5.

Proof of Theorem 1.4. Let $K \subset M, n$ be as in Theorem 1.4. Since $g(M)=g(E(K))$, we have $g(E(n K))=g\left(\#_{i=1}^{n} M\right)$ by Lemma 3.5. 
Note that $g\left(\#_{i=1}^{n} M\right)=n g(M)$ by Haken (Proposition II.10 of Jac80). Hence we have:

$$
\begin{aligned}
t(n K) & =g(E(n K))-1 \\
& =n g(M)-1 \\
& =n(t(K)+1)-1 \\
& =n t(K)+(n-1) .
\end{aligned}
$$

This completes the proof of Theorem 1.4.

\section{EXAMPLE}

Let $K_{m}=K(7,17 ; 10 m-4)$ be the knot introduced by MorimotoSakuma-Yokota in MSY96. That is, $K$ is obtained from the torus knot $K(7,17)$ by adding twists along an unknotted curve $\gamma$ as in Figure 7 .
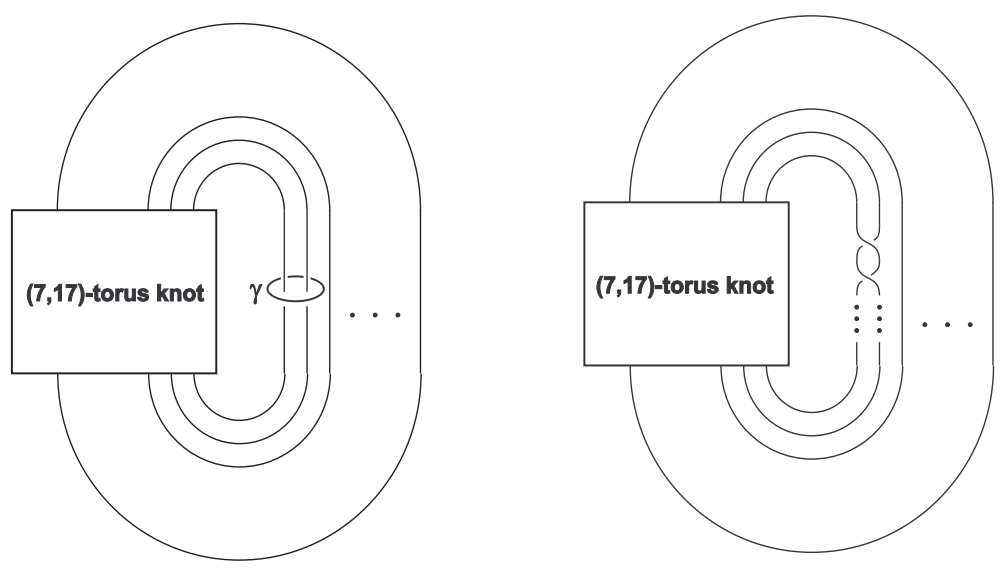

Figure 7

In MSY96] Morimoto-Sakuma-Yokota show that the tunnel number of $K_{m}$ is super additive, i.e., $t\left(K_{m} \# K_{m}\right)=2 t\left(K_{m}\right)+1$; specifically, $t\left(K_{m}\right)=1$ and $t\left(K_{m} \# K_{m}\right)=3$. In particular, $K_{m}$ does not admit a primitive meridian (Proposition 2.1).

We show the following:

Assertion. The knots $K_{m}$ are $(1,2)$-knots; therefore (since $K_{m}$ are super additive) by Proposition [1.7. $K_{m} \# K_{m}$ admits a primitive meridian. 
Proof. $K_{m}$ does not admit a genus 1, 1-bridge presentation, or it would contain a primitive meridian (Proposition 2.4). Hence for the proof of the assertion it suffices to show that $K_{m}$ admits a genus 1, 2-bridge presentation.

Note that $K(7,17)$ is embedded on a genus 1 unknotted torus in $S^{3}$, say $T$. Let $N(T)$ be a regular neighborhood of $T, N(T)=T \times[0,1]$, where $K(7,17) \subset T \times\left\{\frac{1}{2}\right\}$. Here we regard the projection onto the second factor of $T \times[0,1]$ as a height function. Then we can isotope $K(7,17) \cup \gamma$ in $N(T)$ (see Figure 8) so that $K(7,17)=\alpha_{1} \cup \beta_{1} \cup \alpha_{2} \cup \beta_{2}$, where the $\alpha_{i}$ 's and $\beta_{j}$ 's are arcs, each $\alpha_{i}$ and $\beta_{j}$ share exactly one endpoint, and $\alpha_{1} \cap \alpha_{2}=\beta_{1} \cap \beta_{2}=\emptyset(i, j=1,2)$, so that:

(1) $\alpha_{1}$ and $\alpha_{2}$ are monotonic,

(2) $\beta_{1}$ and $\beta_{2}$ are vertical, and-

(3) $\gamma$ is embedded in $T \times\left\{\frac{1}{2}\right\}$ and bounds a disk $D$ in $T \times\left\{\frac{1}{2}\right\}$ such that $D \cap K(7,17)=D \cap\left(\beta_{1} \cup \beta_{2}\right)$ consists of two points, one in $\beta_{1}$ and the other in $\beta_{2}$.
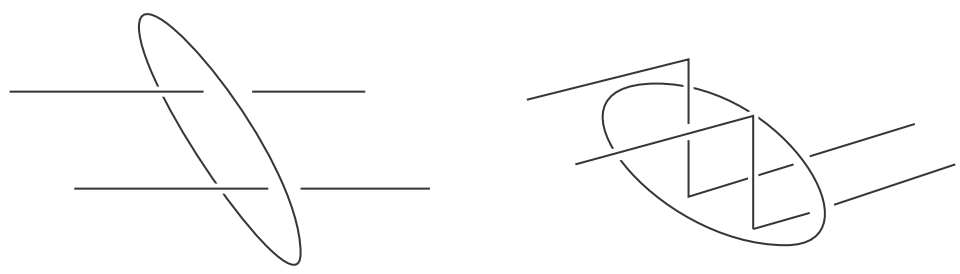

Figure 8

We note that the new position of $K(7,17)$ is a genus 1, 2-bridge presentation. Twisting about $\gamma$ changes only the arcs $\beta_{1}$ and $\beta_{2}$, but after the twist they remain monotonic; this completes the proof of the assertion.

By Theorem 1.2 and Assertion we have that $g r_{t}\left(K_{m}\right) \leq 1 / 2$.

\section{REFERENCES}

[Dol92] H. Doll, A generalized bridge number for links in 3-manifolds, Math. Ann. 294 (1992), no. 4, 701-717. MR 93i:57023

[Hat82] A. E. Hatcher, On the boundary curves of incompressible surfaces, Pacific J. Math. 99 (1982), no. 2, 373-377. MR 83h:57016

[Hem76] John Hempel, 3-Manifolds, Princeton University Press, Princeton, N. J., 1976, Ann. of Math. Studies, No. 86. MR 54 \#3702 
[Jac80] William Jaco, Lectures on three-manifold topology, CBMS Regional Conference Series in Mathematics, vol. 43, American Mathematical Society, Providence, R.I., 1980. MR 81k:57009

[Kob94] Tsuyoshi Kobayashi, A construction of arbitrarily high degeneration of tunnel numbers of knots under connected sum, J. Knot Theory Ramifications 3 (1994), no. 2, 179-186. MR 95g:57011

[Kob01] _ Heegaard splittings of exteriors of two bridge knots, Geom. Topol. 5 (2001), 609-650 (electronic). MR 2002k:57013

[KR] Tsuyoshi Kobayashi and Yo'av Rieck, Heegaard genus of the connected sum of m-small knots, In preparation.

[Kwo94] H-Z Kwong, Streightening tori in heegaard splittings, Ph.D. thesis, UC Santa Barbara, 1994.

[Mor00a] Kanji Morimoto, On the super additivity of tunnel number of knots, Math. Ann. 317 (2000), no. 3, 489-508. MR 2001g:57016

[Mor00b] _ , Tunnel number, connected sum and meridional essential surfaces, Topology 39 (2000), no. 3, 469-485. MR 2001a:57015

[MSY96] Kanji Morimoto, Makoto Sakuma, and Yoshiyuki Yokota, Examples of tunnel number one knots which have the property " $1+1=3$ ", Math. Proc. Cambridge Philos. Soc. 119 (1996), no. 1, 113-118. MR 96i:57007

[Rie00] Yo'av Rieck, Heegaard structures of manifolds in the Dehn filling space, Topology 39 (2000), no. 3, 619-641. MR 2001b:57037

[Sch93] Jennifer Schultens, The classification of Heegaard splittings for (compact orientable surface) $\times S^{1}$, Proc. London Math. Soc. (3) 67 (1993), no. 2, 425-448. MR 94d:57043

[SS00] Martin Scharlemann and Jennifer Schultens, Annuli in generalized Heegaard splittings and degeneration of tunnel number, Math. Ann. 317 (2000), no. 4, 783-820. MR 2001j:57013

Department of Mathematics, Nara Women's University Kitauoya Nishimachi, NARA 630-8506, JaPAN

Department of mathematical Sciences, University of Arkansas, FayetTEVILLE, AR 72701

E-mail address: tsuyoshi@cc.nara-wu.ac.jp

E-mail address: yoav@uark.edu 